\title{
Segmented lordotic angles to assess lumbosacral transitional vertebra on EOS
}

\author{
Domenico Albano ${ }^{1,2}$ (D) Carmelo Messina ${ }^{1,3}$. Angelo Gambino ${ }^{1}$. Martina Gurgitano ${ }^{4}$. Carmelo Sciabica ${ }^{2}$. \\ Giordano Remo Oliveira Pavan ${ }^{5}$. Salvatore Gitto ${ }^{3} \cdot$ Luca Maria Sconfienza ${ }^{1,3}$
}

Received: 22 April 2020 / Revised: 29 June 2020 / Accepted: 4 August 2020 / Published online: 11 August 2020

(c) The Author(s) 2020

\begin{abstract}
Purpose To test the vertical posterior vertebral angles (VPVA) of the most caudal lumbar segments measured on EOS to identify and classify the lumbosacral transitional vertebra (LSTV).

Methods We reviewed the EOS examinations of 906 patients to measure the VPVA at the most caudal lumbar segment (cVPVA) and at the immediately proximal segment (pVPVA), with dVPVA being the result of their difference. Mann-Whitney, Chi-square, and ROC curve statistics were used.

Results 172/906 patients (19\%) had LSTV (112 females, mean age: $43 \pm 21$ years), and 89/172 had type I LSTV (52\%), 42/172 type II (24\%), 33/172 type III (19\%), and 8/172 type IV (5\%). The cVPVA and dVPVA in non-articulated patients were significantly higher than those of patients with LSTV, patients with only accessory articulations, and patients with only bony fusion (all $p<.001$ ). The cVPVA and dVPVA in L5 sacralization were significantly higher than in S1 lumbarization $(p<.001)$. The following optimal cutoff was found: $c$ VPVA of $28.2^{\circ}(\mathrm{AUC}=0.797)$ and dVPVA of $11.1^{\circ}(\mathrm{AUC}=0.782)$ to identify LSTV; cVPVA of $28.2^{\circ}(\mathrm{AUC}=0.665)$ and dVPVA of $8^{\circ}(\mathrm{AUC}=0.718)$ to identify type II LSTV; cVPVA of $25.5^{\circ}$ $(\mathrm{AUC}=0.797)$ and dVPVA of $-7.5^{\circ}(\mathrm{AUC}=0.831)$ to identify type III-IV LSTV; cVPVA of $20.4^{\circ}(\mathrm{AUC}=0.693)$ and dVPVA of $-1.8^{\circ}($ AUC $=0.665)$ to differentiate type II from III-IV LSTV; cVPVA of $17.9^{\circ}(\mathrm{AUC}=0.741)$ and dVPVA of $-4.5^{\circ}(\mathrm{AUC}=0.774)$ to differentiate $\mathrm{L} 5$ sacralization from $\mathrm{S} 1$ lumbarization.

Conclusion The cVPVA and dVPVA measured on EOS showed good diagnostic performance to identify LSTV, to correctly classify it, and to differentiate L5 sacralization from S1 lumbarization.
\end{abstract}

Keywords Lumbar spine $\cdot$ Lumbosacral transitional vertebra $\cdot$ LSTV $\cdot$ EOS $\cdot$ Angles

Domenico Albano

albanodomenico@me.com

1 IRCCS Istituto Ortopedico Galeazzi, Via Riccardo Galeazzi 4, 20161 Milano, Italy

2 Sezione di Scienze Radiologiche, Dipartimento di Biomedicina, Neuroscienze e Diagnostica Avanzata, Università degli Studi di Palermo, Via del Vespro 127, 90127 Palermo, Italy

3 Dipartimento di Scienze Biomediche per la Salute, Università degli Studi di Milano, Via Pascal 36, 20133 Milano, Italy

4 Divisione di Radiologia, IEO Istituto Europeo di Oncologia IRCCS, Via Ripamonti 435, 20141 Milano, Italy

5 Università degli Studi di Milano, Via Festa del Perdono, 7, 20122 Milano, Italy

\section{Introduction}

Transitional anomalies at the lumbosacral junction are quite common being observed in up to one-third of the population. Lumbosacral transitional vertebra (LSTV) is more frequently seen in L5 rather than S1 segment [1]. LSTV has been extensively studied from different perspectives, to understand the underlying genetic factors, to clarify the biomechanical and clinical impact, and to overcome the diagnostic challenges of this condition. The relevance of LSTV is that it can be associated with wrong-level surgery/procedures, degenerative changes of articular facets, and adjacent disks herniation in young patients, with debated association with low back pain [2-4]. Further, LSTV involves also neural arch elements, potentially leading to lumbar spine instability and predisposition to spondylolysis/spondylolisthesis $[5,6]$. According to Castellvi et al. [7], LSTV is classified 
as follows: type I, unilateral (Ia) or bilateral (Ib) enlarged transverse processes; type II, unilateral (IIa) or bilateral (IIb) unfused accessory articulation of the transverse processes with the vertebral below; type III, unilateral (IIIa) or bilateral (IIIb) bony fusion of the transverse processes; type IV, accessory articulation in one side and bony fusion in the other side. For what concerns the controversial association of LSTV with low back pain, it is still unclear whether low back pain is more common or worse in LSTV $[8,9]$. In a large series of patients, Nardo et al. reported significantly higher prevalence of low back pain in type II (73\%) and type IV (66\%) LSTV as compared to patients without LSTV (54\%), with even lower frequency in type I (46\%) and type III (40\%) LSTV [3]. These results were subsequently confirmed by Tang et al. [10], suggesting type II and type IV LSTV as risk factors for low back pain. Hence, a correct LSTV identification has non-negligible impact on surgical and clinical management of patients. In this setting, imaging has well-known limits. In a previous MRI study, more than one-third of LSTV were missed and, in the remaining cases, L5 was defined incorrectly in $60 \%$ of patients [11]. Conventional radiography (CR) is more accurate in both detection and classification of transitional anomalies [12]. Several studies have tested variable vertebral dimensions and angle measurements at the lumbosacral region, mostly on $\mathrm{CR}$, to find the best way to identify and categorize a LSTV [13]. Farshad et al. used the vertical mid- and anterior vertebral angles of the most caudal segments of the lumbar spine to assess patients with LSTV, reporting very high accuracy using the differences of these angles to recognize a LSTV on CR and MRI [14].

In lumbar spine imaging, $\mathrm{CR}$ is routinely used but is burdened by non-negligible projective deformation and dose of ionizing radiations. Recently, the EOS imaging system has been released, which consists of a couple of X-ray tubes and detectors, orthogonal one to the other, capable of simultaneous acquisition of lateral and anteroposterior views of the full spine and lower limbs in standing position, thus providing images without the typical projective deformation of CR. Two-dimensional EOS images can be transformed into three-dimensional spine models giving an accurate 3D representation of spine anatomy that can be used by surgeons for pre-operative planning to obtain an optimal correction of the spine. The correction can include simulating osteotomies, selecting and positioning cages, and planning the length, width, and shape of the spinal rods in 3D images. It allows for 8-10 times reduction of radiation dose, which is quite important especially for pediatric population [15]. EOS is widely used in scoliosis imaging [16, 17], although it is still routinely applied in few countries. But its utility has never been tested in the evaluation of LSTV. Thus, we aimed to test the vertical posterior vertebral angles (VPVA) of the most caudal lumbar segments measured on a large series of patients subjected to EOS system examinations: (i) to identify a LSTV; (ii) to correctly classify a LSTV according to Castellvi grading; and (iii) to distinguish L5 sacralization from S1 lumbarization.

\section{Materials and methods}

\section{Study population}

This retrospective study was approved by our Institutional Review Board (RETRORAD, approved on May 11, 2017, by Ospedale San Raffaele, Milano, Italy) with a waiver for patients' informed consent. This study is concerned with the evaluation of segmented lordotic angles to assess and classify LSTV on EOS system. Hence, we conducted a Picture Archive and Communications System search to include consecutive patients who underwent EOS from September 2017 to January 2018 at our Institution, a tertiary orthopedic surgery center, for several reasons including pre-operative evaluation for spine surgery, scoliosis, lordosis, kyphosis, spondylolysis, spondylolisthesis, spinal stenosis, fractures and non-specific back pain. We excluded patients subjected to previous lumbosacral surgery, follow-up examinations that would have led to duplication of data, and those with gonad protections shields that hindered the evaluation of lumbosacral junction in anteroposterior images. Of 1,348 potentially eligible EOS examinations performed in the index period, 906 were finally included in our series (614 females, 292 males).

\section{EOS system}

Low-dose biplanar (anteroposterior and lateral) X-ray-based images were acquired with the EOS system (EOS Imaging SA, Paris, France) in weight-bearing position with arms and shoulders flexed, hands on cheeks, and free breathing [18]. At least the whole spine, the pelvis, and the femoral heads were included in the images, with digitized images having been simultaneously obtained in coronal and sagittal planes. The EOS system provides spatially calibrated images; thereby, no further calibration was required. Images were acquired with the following standard parameters: $90 \mathrm{kV}$ and $200 \mathrm{~mA}$ for anteroposterior images and $105 \mathrm{kV}$ and $250 \mathrm{~mA}$ for lateral images. Acquisition parameters were adapted to patient habitus when needed.

\section{Images interpretation}

EOS images were first reviewed by a radiologist with 7 years' experience in spine imaging who identified patients with LSTV and classified LSTV according to Castellvi method [7]. Then, similar to a previous study by Farshad 
et al. [14], a radiologist with three years' experience in spine imaging, blinded to Castellvi classification and having access to sagittal images only, measured the segmented lordotic angles using the vertical posterior vertebral angles (VPVA) of the two most caudal lumbar segments and their difference (dVPVA). The segmented lordotic angles were measured on sagittal images at the most caudal segment (that with a fully developed disk extending across the full width of a segment) and at the immediately proximal segment, being defined as cVPVA and pVPVA, respectively. The dVPVA was the result of the difference between the cVPVA and pVPVA.

\section{Statistical analysis}

Continuous variables are reported as mean \pm standard deviation. Statistical differences between patients with and those without LSTV, as well as between different types of LSTV, were compared using Mann-Whitney U test. Specifically, we evaluated the differences between non-articulated patients (non-LSTV + type I LSTV), patients with unfused accessory articulation (type II LSTV), and patients with osseous fusion (type III-IV LSTV). Frequencies were compared using the Chi-square test. Receiver operating characteristic (ROC) curve analysis and calculation of the area under the ROC curve (AUC) were used to calculate the diagnostic performance of cVPVA and dVPVA as diagnostic tools. A $P$ value $<0.05$ was considered statistically significant [19]. The SPSS software (v. 26, IBM, Armonk, New York, NY) was used for statistical analysis.

\section{Results}

Of 906 patients included in our series, 172 (19\%) had LSTV (112 females, 60 males; mean age: $43 \pm 21$ years). The presence of LSTV was slightly but not significantly ( $p=0.685)$ more frequent in males $(60 / 292,21 \%)$ than in females $(112 / 614,18 \%)$. Eighty-nine out of 172 patients had type I LSTV (52\%), 42/172 had type II (24\%), 33/172 had type III (19\%), and 8/172 had type IV (4\%). The mean cVPVA, pVPVA, and dVPVA were $32.3^{\circ} \pm 10.2^{\circ}$, $16.2^{\circ} \pm 7.2^{\circ}$, and $16^{\circ} \pm 14.1^{\circ}$ in non-articulated patients, $22.2^{\circ} \pm 12.7^{\circ}, 20.6^{\circ} \pm 10.8^{\circ}$, and $1.6^{\circ} \pm 19.7^{\circ}$ in patients with unfused accessory articulation, and $14.9^{\circ} \pm 10.9^{\circ}$, $24.8^{\circ} \pm 11^{\circ}$, and $-9.9^{\circ} \pm 19.3^{\circ}$ in fused patients. The values of cVPVA and dVPVA of non-articulated patients were significantly higher than those of patients with LSTV, patients with only unfused accessory articulations, and patients with only bony fusion (all with $p<0.001$ ). These measures were also significantly higher in patients with unfused accessory articulations when compared with patients with bony fusion ( $p=0.002$ for cVPVA, $p=0.010$ for dVPVA). The cVPVA and dVPVA of patients with L5 sacralization were higher than those of patients with S1 lumbarization; these differences were statistically significant $(p<0.001)$ when all types of Castellvi were included, while it was not significant when only patients with unfused accessory articulation and bony fusion (types II-III-IV) were analyzed ( $p=0.211$ and $p=0.140$, respectively). Full data and statistical comparisons of mean cVPVA, pVPVA, and dVPVA values are reported in Tables 1 and 2. Figures 1 and 2 show two cases from our study population.

Full ROC curve analysis is reported in Table 3. Specifically, the following optimal cutoff values were found:

cVPVA of $28.2^{\circ}(\mathrm{AUC}=0.797)$ and dVPVA of $11.1^{\circ}$ $(\mathrm{AUC}=0.782)$ to identify LSTV;

cVPVA of $28.2^{\circ}(\mathrm{AUC}=0.665)$ and dVPVA of $8^{\circ}$ $(\mathrm{AUC}=0.718)$ to identify type II LSTV;

cVPVA of $25.5^{\circ}(\mathrm{AUC}=0.797)$ and dVPVA of $-7.5^{\circ}$ (AUC $=0.831)$ to identify type III-IV LSTV;

cVPVA of $20.4^{\circ}$ (AUC $=0.693$ ) and dVPVA of $-1.8^{\circ}$ $(\mathrm{AUC}=0.665)$ to differentiate type II LSTV from type III-IV LSTV;

cVPVA of $17.9^{\circ}(\mathrm{AUC}=0.741)$ and dVPVA of $-4.5^{\circ}$ $(\mathrm{AUC}=0.774)$ to differentiate L5 sacralization from S1 lumbarization including all Castellvi types.
Table 1 The mean \pm standard deviation of cVPVA, pVPVA, dVPVA of 906 patients on the basis of LSTV classification according to Castellvi

\begin{tabular}{llllrr}
\hline & No. of patients & Gender & \multicolumn{1}{l}{ cVPVA } & \multicolumn{1}{l}{ pVPVA } & \multicolumn{1}{c}{ dVPVA } \\
\hline No LSTV & 734 & $230 \mathrm{~m}, 502 \mathrm{f}$ & $32.4^{\circ} \pm 10.1^{\circ}$ & $16.2^{\circ} \pm 7.1^{\circ}$ & $16.2^{\circ} \pm 13.7^{\circ}$ \\
Castellvi I & 89 & $37 \mathrm{~m}, 52 \mathrm{f}$ & $30.8^{\circ} \pm 11.6^{\circ}$ & $16.1^{\circ} \pm 7.7^{\circ}$ & $14.7^{\circ} \pm 16.5^{\circ}$ \\
Castellvi II & 42 & $13 \mathrm{~m}, 29 \mathrm{f}$ & $22.2^{\circ} \pm 12.7^{\circ}$ & $20.6^{\circ} \pm 10.8^{\circ}$ & $1.6^{\circ} \pm 19.7^{\circ}$ \\
Castellvi III & 33 & $8 \mathrm{~m}, 25 \mathrm{f}$ & $13.9^{\circ} \pm 10.9^{\circ}$ & $25^{\circ} \pm 12.1^{\circ}$ & $-11.1^{\circ} \pm 20.3^{\circ}$ \\
Castellvi IV & 8 & $2 \mathrm{~m}, 6 \mathrm{f}$ & $18.8^{\circ} \pm 10.6^{\circ}$ & $23.9^{\circ} \pm 5^{\circ}$ & $-5^{\circ} \pm 14.1^{\circ}$ \\
\hline
\end{tabular}

$\mathrm{cVPVA}=$ caudal vertical posterior vertebral angle; $\mathrm{pVPVA}=$ proximal vertical posterior vertebral angle; $\mathrm{dVPVA}=$ difference of vertical posterior vertebral angles; LSTV = lumbosacral transitional vertebra 
Table 2 All statistical comparisons of mean cVPVA, pVPVA, and dVPVA values

\begin{tabular}{llll}
\hline & cVPVA & pVPVA & dVPVA \\
\hline No LSTV + Cast I & $32.3^{\circ} \pm 10.2^{\circ}$ & $16.2^{\circ} \pm 7.2^{\circ}$ & $16^{\circ} \pm 14.1^{\circ}$ \\
Cast II-III-IV & $18.6^{\circ} \pm 12.3^{\circ}$ & $22.7^{\circ} \pm 11^{\circ}$ & $-4.1^{\circ} \pm 20.2^{\circ}$ \\
$P$ & $<.001$ & $<.001$ & $<.001$ \\
No LSTV + Cast I & $32.3^{\circ} \pm 10.2^{\circ}$ & $16.2^{\circ} \pm 7.2^{\circ}$ & $16^{\circ} \pm 14.1^{\circ}$ \\
Cast II & $22.2^{\circ} \pm 12.7^{\circ}$ & $20.6^{\circ} \pm 10.8^{\circ}$ & $1.6^{\circ} \pm 19.7^{\circ}$ \\
$P$ & $<.001$ & .072 & $<.001$ \\
No LSTV +Cast I & $32.3^{\circ} \pm 10.2^{\circ}$ & $16.2^{\circ} \pm 7.2^{\circ}$ & $16^{\circ} \pm 14.1^{\circ}$ \\
Cast III-IV & $14.9^{\circ} \pm 10.9^{\circ}$ & $24.8^{\circ} \pm 11^{\circ}$ & $-9.9^{\circ} \pm 19.3^{\circ}$ \\
$P$ & $<.001$ & $<.001$ & $<.001$ \\
Cast II & $22.2^{\circ} \pm 12.7^{\circ}$ & $20.6^{\circ} \pm 10.8^{\circ}$ & $1.6^{\circ} \pm 19.7^{\circ}$ \\
Cast III-IV & $14.9^{\circ} \pm 10.9^{\circ}$ & $24.8^{\circ} \pm 11^{\circ}$ & $-9.9^{\circ} \pm 19.3^{\circ}$ \\
$P$ & .002 & .076 & $.010^{\circ}$ \\
L5 sacralization (Cast I-II-III-IV) & $26.4^{\circ} \pm 12.9^{\circ}$ & $18.3^{\circ} \pm 10.1^{\circ}$ & $8.1^{\circ} \pm 20.3^{\circ}$ \\
S1 lumbarization (Cast I-II-III-IV) & $14.9^{\circ} \pm 12.4^{\circ}$ & $25.7^{\circ} \pm 6.7^{\circ}$ & $-10.8^{\circ} \pm 14.5^{\circ}$ \\
$P$ & $<.001$ & $<.001$ & $<.001$ \\
L5 sacralization (Cast II-III-IV) & $19.6^{\circ} \pm 12.2^{\circ}$ & $21.8^{\circ} \pm 12.1^{\circ}$ & $-2.2^{\circ} \pm 21.8^{\circ}$ \\
S1 lumbarization (Cast II-III-IV) & $15.6^{\circ} \pm 12.2^{\circ}$ & $25.2^{\circ} \pm 6.5^{\circ}$ & $-9.7^{\circ} \pm 13.8^{\circ}$ \\
$P$ & .211 & .196 & .140 \\
\hline
\end{tabular}

cVPVA = caudal vertical posterior vertebral angle; $\mathrm{pVPVA}=$ proximal vertical posterior vertebral angle; dVPVA = difference of vertical posterior vertebral angles; LSTV=lumbosacral transitional vertebra; Cast $=$ Castellvi type; $P=$ statistical significance after Mann-Whitney $U$ test; italic indicates all significant differences $(p<.002)$

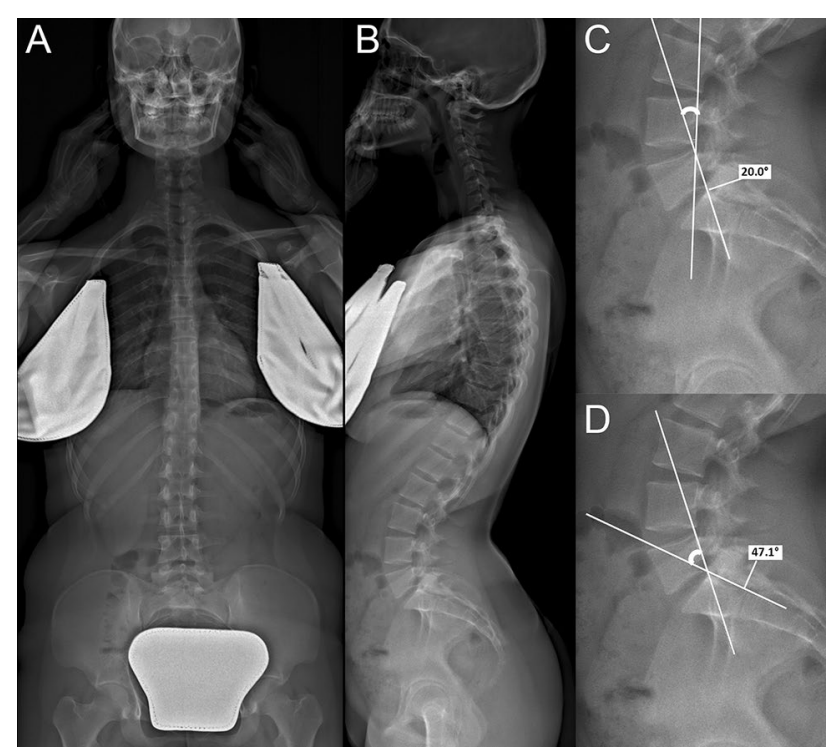

Fig. 1 EOS examination of a 32-year-old female patient without LSTV. Coronal (a) and sagittal (b) EOS examination of the full spine. In detail, pVPVA of $20.0^{\circ}$ (c) and cVPVA of $47.1^{\circ}$ (d) on sagittal views of the lumbosacral junction. The dVPVA $=$ cVPVA$\mathrm{pVPVA}=47.1^{\circ}-20.0^{\circ}=27.1^{\circ}$

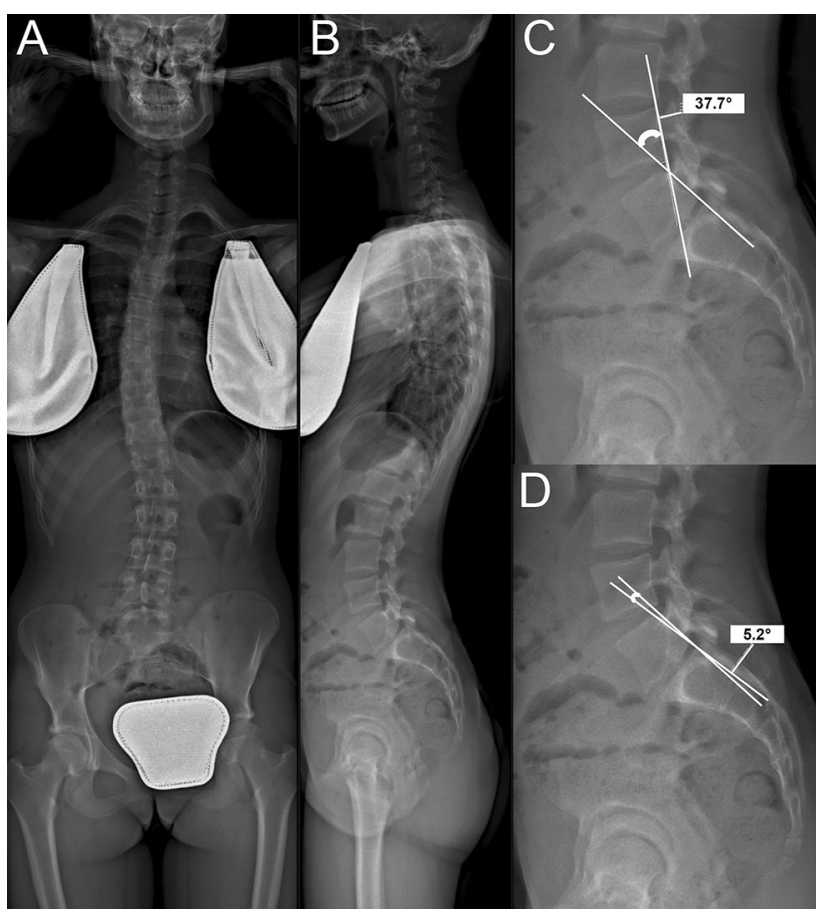

Fig. 2 EOS examination of a 25-year-old female patient without LSTV. Coronal (a) and sagittal (b) EOS examination of the full spine. In detail, pVPVA of $37.7^{\circ}$ (c) and cVPVA of $5.2^{\circ}$ (d) on sagittal views of the lumbosacral junction. The dVPVA $=$ cVPVA$\mathrm{pVPVA}=5.2^{\circ}-37.7^{\circ}=-32.5^{\circ}$ 
Table 3 Full ROC curve analysis

\begin{tabular}{|c|c|c|c|c|c|c|c|c|}
\hline & \multicolumn{4}{|l|}{ cVPVA } & \multicolumn{4}{|l|}{ dVPVA } \\
\hline & Cutoff & $\begin{array}{l}\text { Sensitiv- } \\
\text { ity (\%) }\end{array}$ & $\begin{array}{l}\text { Specific- } \\
\text { ity }(\%)\end{array}$ & Accuracy $(\%)$ & Cutoff & $\begin{array}{l}\text { Sensitiv- } \\
\text { ity (\%) }\end{array}$ & $\begin{array}{l}\text { Specific- } \\
\text { ity (\%) }\end{array}$ & Accuracy $(\%)$ \\
\hline LSTV II-III-IV vs. non-LSTV + LSTV I & $28.2^{\circ}$ & 77 & 67 & 80 & $11.1^{\circ}$ & 72 & 67 & 78 \\
\hline LSTV III-IV vs. non-LSTV + LSTV I & $25.5^{\circ}$ & 71 & 79 & 80 & $-7.5^{\circ}$ & 71 & 92 & 83 \\
\hline LSTV II vs. non-LSTV + LSTV I & $28.2^{\circ}$ & 64 & 71 & 66 & $8^{\circ}$ & 67 & 66 & 72 \\
\hline LSTV II vs. LSTV III & $20.4^{\circ}$ & 71 & 57 & 69 & $-1.8^{\circ}$ & 71 & 57 & 66 \\
\hline L5 sacralization vs. S1 lumbarization & $17.9^{\circ}$ & 73 & 75 & 74 & $-4.5^{\circ}$ & 77 & 73 & 77 \\
\hline
\end{tabular}

$\mathrm{cVPVA}=$ caudal vertical posterior vertebral angle; $\mathrm{pVPVA}=$ proximal vertical posterior vertebral angle; $d V P V A=$ difference of vertical posterior vertebral angles; LSTV = lumbosacral transitional vertebra

\section{Discussion}

Our main finding was the good diagnostic performance (accuracy around 80\%) of cVPVA and dVPVA to identify the LSTV, with accuracies ranging from 66 to $83 \%$ to correctly classify a LSTV according to Castellvi and to differentiate L5 sacralization from S1 lumbarization.

In our study, LSTV had a prevalence of $19 \%$ and was slightly more frequent in men, which is in line with previous studies, although high variability exists in the reported frequency of LSTV. In a series of 4,636 patients, Nardo et al. reported a prevalence of $18 \%$ with significantly higher frequency in men $(28 \%)$ than in women (11\%) [4]. Delport et al. found LSTV in 30\% of patients, although their sample size was smaller ( $n=300$ patients) [20]. Anyway, this condition is quite common in the general population and needs to be considered in patients with low back pain, especially prior to spine injections or surgery [21].

In this setting, the main challenge is the detection and classification of LSTV. Anteroposterior CR is the reference standard for LSTV detection with accuracy of $76-84 \%$, while the accuracy in the classification of LSTV is lower (53-58\%) [12]. Sagittal radiograph shows the typical "squaring" of the LSTV and lower height of the transitional disk [22]. The diagnostic performance of MRI is even lower due to the absence of coronal images in most standard protocols of the lumbar spine. EOS examination enables to easily identify LSTV since whole-spine coronal images, which we used as reference to classify our patients, allow for precise identification of both numeric and morphological vertebral variations and to correctly identify L1 vertebral body by differentiating hypoplastic true ribs from large transverse processes at the thoracolumbar junction [23]. This can be done with minimal radiation exposure, avoiding the magnification error of $\mathrm{CR}$, and in weight-bearing position therefore obtaining essential information about spinal balance [24, 25]. Once having classified our patients, we tested the diagnostic performance of the posterior vertebral angles of the two most caudal lumbar segments (cVPVA and pVPVA) and their difference (dVPVA). Similarly, Farshad et al. used the vertical mid- and anterior vertebral angles reporting sensitivity of 94-100\% and specificity of 74-89\% using the differences of these angles to identify LSTV on CR and MRI [14]. Specifically, they found that the difference of the most caudal angles of $10^{\circ}$ yielded a sensitivity of $100 \%$ and a specificity of $89 \%$ on MRI and a sensitivity of $94 \%$ and a specificity of $74 \%$ on sagittal radiograph to identify type III-IV LSTV. As in their study, we observed the greatest cVPVA in non-articulated patients with a progressive decrease in patients with unfused accessory articulation and bony fusion, associated with progressive increase in pVPVA. Of course, a progressive decrease in dVPVA was also found with differences having shown negative values in patients with bony fusion (mean dVPVA of $-9.9 \pm 19.3^{\circ}$ in type III-IV LSTV). A dVPVA of $11.1^{\circ}$ yielded sensitivity of $72 \%$ and specificity of $67 \%$ to differentiate LSTV from non-articulated patients. This is related to the lack of sharp angulations at the lumbosacral junction in patients with transitional anomalies and increased lumbar lordotic curvature cranial to the LSTV [12]. Our measurements, compared with the radiographic results of Farshad et al., yielded slightly lower values of accuracy, with lower sensitivity $(71 \%)$ but higher specificity $(92 \%)$ using $-7.5^{\circ}$ as optimal dVPVA to identify type III-IV LSTV. If we had used $10^{\circ}$ as optimal cutoff, as done by Farshad et al., we would have reached sensitivity of $71 \%$ but specificity of $86 \%$. We also found that an optimal cVPVA of $28.2^{\circ}$ had sensitivity and specificity of $77 \%$ and $67 \%$ to identify a LSTV and $64 \%$ and $66 \%$ to differentiate type II LSTV from non-articulated patients. These results show how these measurements may increase the diagnostic performance of standard sagittal radiograph in the classification of LSTV, but underline a possible drawback of the measurements proposed by Farshad et al. Indeed, the measurements of the posterior vertebral angles are very similar to those obtained at the anterior side and mid-portion of the vertebral bodies in sagittal views by 
Farshad et al. who probably evaluated non-weight-bearing CR (although it is not clear), but the accuracy of our measures could have been slightly lower due to the acquisition in weight-bearing position.

Another issue is the recognition of LSTV as L5 or S1, since transitional anomalies may lead to wrong-level surgery and procedures. To correctly number a LSTV, several indirect signs related to the position of paraspinal structures have been proposed, including the insertion of iliolumbar ligaments, the position of aortic bifurcation, inferior vena cava confluence, right renal artery, celiac trunk, and superior mesenteric artery, although the position of these structures varies widely from one person to another and with increasing age [23]. We even tried to understand whether the segmented lordotic angles could be helpful to differentiate L5 sacralization from S1 lumbarization in sagittal views. We found significantly higher cVPVA and dVPVA, and significantly lower pVPVA, in patients with L5 sacralization than in those with $\mathrm{S} 1$ lumbarization. The cVPVA and dVPVA of $17.9^{\circ}$ and $-4.5^{\circ}$ reached accuracy of $74 \%$ and $77 \%$, respectively, with values of sensitivity and specificity always higher than $70 \%$. Thus, it seems that when the LSTV is L5, the junction lordotic angle is higher than in case of S1 lumbarization with an exaggerated lordotic angle in the immediately cranial segment (pVPVA). This could be helpful to correctly number the LSTV when anteroposterior radiography is not univocal or when dealing with MRI without coronal images. However, these data need to be confirmed by studies on MRI and non-weight-bearing $\mathrm{CR}$.

Some limitations have to be considered. First, our series included a little number of type IV LSTV, but it was expected being a very rare condition. Then, we did not consider the correlation of our measurements with the sacral inclination angle, sacral slope, pelvic incidence, and pelvic tilt. However, although we could obtain these values on EOS examination, we decided to test angles which are easily and quickly obtained also on standard CR and MRI as for a potential use in daily clinical practice. Last, we did not compare our values with those obtained in CR and MRI examination, which should be the subject of future studies.

In conclusion, we reported the good diagnostic performance of posterior vertebral angles of the two most caudal segments and their difference to identify LSTV, to correctly classify it, and to differentiate L5 sacralization from S1 lumbarization. Further studies are needed to confirm our results and to test the application of these measurements in non-weight-bearing CR and MRI.

Funding This research did not receive any specific grant from funding agencies in the public, commercial, or not-for-profit sectors. Open access funding provided by Università degli Studi di Palermo within the CRUI-CARE Agreement.

\section{Compliance with ethical standards}

Conflict of interest The authors declare that they have no conflict of interest.

Ethics approval and consent to participate All procedures performed in studies involving human participants were in accordance with the ethical standards of the institutional and/or national research committee and with the 1964 Helsinki Declaration and its later amendments or comparable ethical standards. This retrospective study was approved by our Institutional Review Board (Ospedale San Raffaele, Milano, Italy) with a waiver for patients' informed consent. This study has been conducted according to the principles expressed in the Declaration of Helsinki.

Open Access This article is licensed under a Creative Commons Attribution 4.0 International License, which permits use, sharing, adaptation, distribution and reproduction in any medium or format, as long as you give appropriate credit to the original author(s) and the source, provide a link to the Creative Commons licence, and indicate if changes were made. The images or other third party material in this article are included in the article's Creative Commons licence, unless indicated otherwise in a credit line to the material. If material is not included in the article's Creative Commons licence and your intended use is not permitted by statutory regulation or exceeds the permitted use, you will need to obtain permission directly from the copyright holder. To view a copy of this licence, visit http://creativecommons.org/licenses/by/4.0/.

\section{References}

1. Apazidis A, Ricart PA, Diefenbach CM, Spivak JM (2011) The prevalence of transitional vertebrae in the lumbar spine. Spine $\mathrm{J}$ 11:858-862. https://doi.org/10.1016/j.spinee.2011.08.005

2. Malanga GA, Cooke PM (2004) Segmental anomaly leading to wrong level disc surgery in cauda equina syndrome. Pain Physician 7:107-110

3. Luoma K, Vehmas T, Raininko R, Luukkonen R, Riihimäki H (2004) Lumbosacral transitional vertebra: relation to disc degeneration and low back pain. Spine 29:200-205. https://doi. org/10.1097/01.BRS.0000107223.02346.A8

4. Nardo L, Alizai H, Virayavanich W et al (2012) Lumbosacral transitional vertebrae: association with low back pain. Radiology 265:497-503. https://doi.org/10.1148/radiol.12112747

5. Mahato NK (2011) Pedicular anatomy of the first sacral segment in transitional variations of the lumbo-sacral junction. Spine 36(18):E1187-E1192. https://doi.org/10.1097/BRS.0b013e3182 06bdf7

6. Mahato NK (2013) Pars inter-articularis and laminar morphology of the terminal lumbar vertebra in lumbosacral transitional variations. N Am J Med Sci 5:357-361. https://doi.org/10.4103/19472714.114167

7. Castellvi AE, Goldstein LA, Chan DP (1984) Lumbosacral transitional vertebrae and their relationship with lumbar extradural defects. Spine 9:493-495. https://doi.org/10.1097/00007632198407000-00014

8. Quinlan JF, Duke D, Eustace S (2006) Bertolotti's syndrome. A cause of back pain in young people. J Bone Joint Surg Br 88:1183-1186. https://doi.org/10.1302/0301-620X.88B9.17211 
9. Yavuz U, Bayhan AI, Beng K, Emrem K, Uzun M (2012) Low back complaints worse, but not more frequent in subjects with congenital lumbosacral malformations: a study on 5000 recruits. Acta Orthop Belg 78:668-671

10. Tang M, Yang XF, Yang SW et al (2014) Lumbosacral transitional vertebra in a population-based study of 5860 individuals: prevalence and relationship to low back pain. Eur J Radiol 83:16791682. https://doi.org/10.1016/j.ejrad.2014.05.036

11. Tokgoz N, Ucar M, Erdogan AB, Kilic K, Ozcan C (2014) Are spinal or paraspinal anatomic markers helpful for vertebral numbering and diagnosing lumbosacral transitional vertebrae? Korean J Radiol 15:258-266. https://doi.org/10.3348/kjr.2014.15.2.258

12. Jancuska JM, Spivak JM, Bendo JA (2015) A review of symptomatic lumbosacral transitional vertebrae: Bertolotti's syndrome. Int J Spine Surg 9:42. https://doi.org/10.14444/2042

13. Mahato NK (2011) Disc spaces, vertebral dimensions, and angle values at the lumbar region: a radioanatomical perspective in spines with L5-S1 transitions: clinical article. J Neurosurg Spine 15:371-379. https://doi.org/10.3171/2011.6.SPINE11113

14. Farshad M, Aichmair A, Hughes AP, Herzog RJ, FarshadAmacker NA (2013) A reliable measurement for identifying a lumbosacral transitional vertebra with a solid bony bridge on a single-slice midsagittal MRI or plain lateral radiograph. Bone Joint J 95:1533-1537. https://doi.org/10.1302/0301-620X.95B11 .32331

15. Girdler S, Cho B, Mikhail CM, Cheung ZB, Maza N, Kang-Wook Cho $S$ (2020) Emerging techniques in diagnostic imaging for idiopathic scoliosis in children and adolescents: a review of the literature. World Neurosurg 136:128-135. https://doi.org/10.1016/j. wneu.2020.01.043

16. Redaelli A, Langella F, Dziubak M et al (2020) Useful and innovative methods for the treatment of postoperative coronal malalignment in adult scoliosis: the "kickstand rod" and "tie rod" procedures. Eur Spine J 29:849-859. https://doi.org/10.1007/s0058 6-019-06285-7

17. Berjano P, Cecchinato R, Sinigaglia A et al (2015) Anterior column realignment from a lateral approach for the treatment of severe sagittal imbalance: a retrospective radiographic study.
Eur Spine J 24(Suppl 3):433-438. https://doi.org/10.1007/s0058 6-015-3930-1

18. Kaneko K, Aota Y, Sekiya T, Yamada K, Saito T (2016) Validation study of arm positions for evaluation of global spinal balance in EOS imaging. Eur J Orthop Surg Traumatol 26:725-733. https ://doi.org/10.1007/s00590-016-1813-8

19. Di Leo G, Sardanelli F (2020) Statistical significance: $p$ value, 005 threshold, and applications to radiomics-reasons for a conservative approach. Eur Radiol Exp 4:18. https://doi.org/10.1186/s4174 7-020-0145-y

20. Delport EG, Cucuzzella TR, Kim N, Marley J, Pruitt C, Delport AG (2006) Lumbosacral transitional vertebrae: incidence in a consecutive patient series. Pain Physician 9:53-56

21. Matson DM, Maccormick LM, Sembrano JN, Polly DW (2020) Sacral dysmorphism and lumbosacral transitional vertebrae (LSTV) review. Int J Spine Surg 14(1):14-19. https://doi. org/10.14444/6075

22. Hughes RJ, Saifuddin A (2004) Imaging of lumbosacral transitional vertebrae. Clin Radiol 59:984-991. https://doi. org/10.1016/j.crad.2004.02.019

23. Carrino JA, Campbell PD Jr, Lin DC et al (2011) Effect of spinal segment variants on numbering vertebral levels at lumbar MR imaging. Radiology 259:196-202. https://doi.org/10.1148/radio 1.11081511

24. Melhem E, Assi A, El Rachkidi R, Ghanem I (2016) EOS(®) biplanar X-ray imaging: concept, developments, benefits, and limitations. J Child Orthop 10:1-14. https://doi.org/10.1007/s1183 2-016-0713-0

25. Dubousset J, Charpak G, Dorion I et al (2005) A new 2D and 3D imaging approach to musculoskeletal physiology and pathology with low-dose radiation and the standing position: the EOS system. Bull Acad Natl Med 189:287-297

Publisher's Note Springer Nature remains neutral with regard to jurisdictional claims in published maps and institutional affiliations. 